\title{
Surface Plasmon Enhancement Effect and Its Application to Near-Field Optical Recording
}

\author{
J. Fujikata, T. Ishi, H. Yokota, K. Kato, M. Yanagisawa, M. Nakada, K. Ishihara, and K. Ohashi \\ T. Thio ${ }^{* 1)}$ and R. A. Linke ${ }^{\left.{ }^{2}\right)}$ \\ Fundamental and Environmental Res. Labs., NEC Corporation, 34 Miyukigaoka, Tsukuba, Ibaraki 305 -8501, Japan \\ "NEC Laboratories America, Inc., 4 Independence Way, Princeton, New Jersey 08540, USA
}

We studied on enhanced photon-tunneling and the near-field enhancement effect for a nano-scale aperture with a concentric surface plasmon resonance structure. About two-orders larger light transmission and an enhanced near-field were achieved by optimizing the grating structures. This enhanced near-field was applied to optical recording, and about $100-\mathrm{nm}$ recorded patterns were successfully made on phase change media. This surface plasmon optics would open up the ultra-high-density recording technology.

Key words: surface plasmon, photon tunneling, near-field enhancement, near-field optical recording, phase change media

\section{Introduction}

Near-field optical recording methods are a key technology for the ultra-high-density recording. ${ }^{1-2)}$ To realize nano-scale optical recording beyond the diffraction limit, a near-field optical fiber probe and a pyramidal $\mathrm{Si}$ probe recording methods have been proposed, and recording of a small bit has been demonstrated. ${ }^{1)-3)}$ However, a near-field optical probe with a subwavelength aperture had remarkably low efficiency of throughput power, and the very high incident power and the long pulse time were reported to be necessary to record a small bit on the recording media.

As for subwavelength apertures, no optical transmission mode exists and an electric field in the hole usually evanescently decays with aperture length. In this case, transmission light intensity varies with $\left.d^{6} / \lambda^{4} .4\right)$ Here, $d$ is the aperture diameter, and $\lambda$ is the incident light wavelength.

Recently, we have reported that a subwavelength single aperture with a concentric grating structure (bull's eye structure) realizes a beaming light as well as a high light transmission. ${ }^{5-8)}$ This surprising discovery has attracted much interest in physics and numerous potential applications in optics and photonics.

For the subwavelength aperture with arrayed structures, the transmission light enhancement effect has been theoretically analyzed by many groups, and has been explained by way of a surface plasmon involved process or by a diffraction theory of metal grating. ${ }^{9)-16)}$ But the transmission enhancement and near-field enhancement effect for the bull's eye structure have not been analyzed in detail.

In addition to these fundamental interests, this

*1) present address: Arinna LLC, 79 Dempsey Avenue, Princeton NJ 08540, USA

*2) present address: Optical Society of America, 2010 Massachusetts Ave NW, Washington DC 20036, USA transmission enhancement effect has potential applications in subwavelength photolithography, near-field optical recording, wavelength-tunable filters, optical modulators, and flat displays, and so on. As for near-field optical recording, a highly enhanced power throughput and a nano-sized optical spot is necessary to form a small pattern on the recording media.

In this paper, we report on enhanced near-field technology by surface plasmon resonance and its application to near-field optical recording.

\section{Experiment}

The bull's eye structure we used had a single aperture of $100-150 \mathrm{~nm}$ in diameter with a concentric grating structure with a $600-\mathrm{nm}$ period.

The process for fabricating the samples started with a $\mathrm{SiO}_{2}$ wafer substrate. $\mathrm{A} \mathrm{SiO}_{2}$ surface layer was patterned into concentric grating structure s with a $600-\mathrm{nm}$ period by focused ion beam (FIB) etching. After fabricating the grating structures on the $\mathrm{SiO}_{2}$ substrate, a silver metal layer with a thickness of 200-300 nm was deposited by ion beam sputtering. At the end, a single $100-150 \mathrm{~nm}$ diameter aperture was made in the center of the concentric grating structure in the silver metal with FIB etching. To investigate the role of the diele ctric properties of the surface metal, a Ta metal layer was coated on the silver layer. The dielectric properties of the $\mathrm{Ag}$ and $\mathrm{Ta}$ layers were $\varepsilon_{\mathrm{Ag}}=-17.24+0.50 \mathrm{i}$ and $\varepsilon_{\mathrm{Ta}}=-4.27+24.63 \mathrm{i}$ at a wavelength of $630 \mathrm{~nm}$ obtained by measurements of variable angle spectroscopic ellipsometry. This indicated that the Ta layer was much more optically lossy than the Ag layer.

Zero-order transmission spectra were recorded, using a confocal microscope coupled to a charge-coupled-device (CCD) spectroscope. The intensity profile and spectra for the optical near-field were obtained with a shear-force-mode near-field scanning optical microscope (NSOM) coupled with a CCD spectroscope and a photomultiplier system, in which a sharpened opt ical fiber probe with a 100-150-nm-diameter aperture was scanned at about a $10-30-\mathrm{nm}$ height above the sample surface. In our experiment, the samples were illuminated from the substrate side, and zero-order transmission spectra exhibited a similar profile with illumination from the air side. Th is coincided with Ebbesen's results that the in- and out-coupling mechanism between light and the corrugated metal surface were equivalent. ${ }^{6}$ )

Near-field optical recording was examined using 
(a)

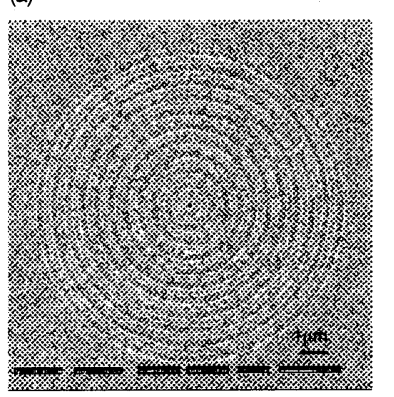

Fig. 1 (a) Horizontal and (b) cross-sectional images of grating structure by scanning ion microscope.

sputtered Ge-Sb-Te phase change media having a GeTe-rich pseudo binary composition. ${ }^{17)}$ The crystallization temperature was between 150 and 200 degrees. The media structure was $15 \mathrm{~nm}$ of $\mathrm{Ge}-\mathrm{Sb}-\mathrm{Te}$ on the $\mathrm{SiO}_{2}$ substrate which was overcoated by $10 \mathrm{~nm}$ of a protective $\mathrm{SiO}_{2}$ layer. Input laser power was $3 \mathrm{~mW}$ and pulse-modulated at a period of $50-500 \mu \mathrm{sec}$.

\section{Results and discussions}

\subsection{Enhanced light transmission effect}

Dispersion and photonic band structures of surface plasmon polaritons (SPP) in the gratings of sinusoidal shape have experimentally been reported, and recently many groups have made analytical reports on photonic band structures of grating coupled SPP. ${ }^{18)} \mathrm{We}$ investigated whether grating coupled SPP contributed to photon tunneling th rough a single aperture with various concentric grating structures. The horizontal and cross sectional images of grating structure by scanning ion microscope (SIM) was shown in Fig. 1, which are like sinusoidal grating structure $\mathrm{s}$.

The dispersion relation of SPP for the p-polarization incident can be written as

$$
k_{S P}=\omega / c\left(\varepsilon_{m} \varepsilon_{d} / \varepsilon_{m}+\varepsilon_{d}\right)^{1 / 2} .
$$

Here, $\varepsilon_{\mathrm{m}}$ and $\varepsilon_{\mathrm{d}}$ are the dielectric constants of the metal and dielectric medium. ${ }^{19)}$ The effective refractive index of an air/Ag interface is estimated to be about 1.03. In this study, the SPP resonance wavelength $\left(\lambda_{\mathrm{SP}}\right)$ at the air/ $\mathrm{Ag}$ interface was about $620 \mathrm{~nm}$ for a grating period of 600

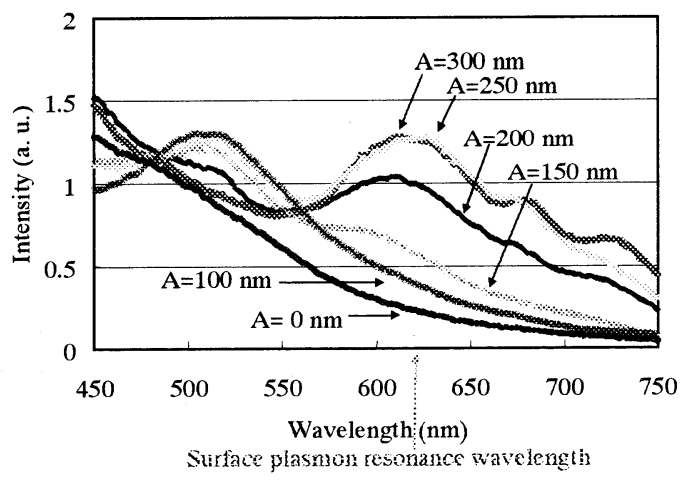

Fig. 2 Dependence of transmission-light spectroscopy on grating amplitude.

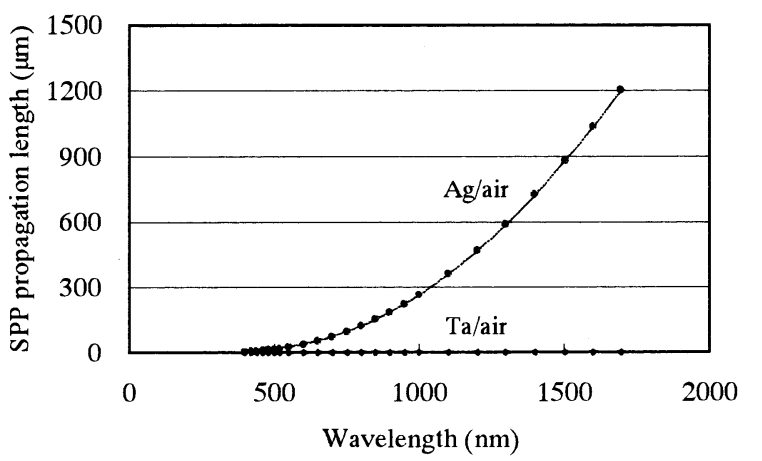

Fig. 3 Estimated SPP propagation length at $\mathrm{Ag} / \mathrm{air}$ and $\mathrm{Ta} /$ air interfaces.

nm.

Figure 2 shows the dependence of transmission light spectroscopy on grating amplitude A for these sinusoidal structures. A transmission peak appeared in the spectrum with increasing grating amplitude and saturated at around $250 \mathrm{~nm}$. The diffraction grating coupling of SPP enhances optical transmission at around the $620-630-\mathrm{nm}$ wavelength.

To study the role of SPP resonance, surface feature effect of silver metal was investigated by coating Ta layers on silver layers. Figure 3 shows the estimated SPP propagation length $\left(\mathrm{L}_{\mathrm{SPP}}\right)$ at the metal/air interface. $\mathrm{L}_{\mathrm{SPP}}$ was estimated as follows, ${ }^{19}$

$$
L_{S P P}=c / \omega\left\{\left(\varepsilon_{m}{ }^{\prime}+1\right) / \varepsilon_{m}\right\}^{3 / 2} \cdot\left(\varepsilon_{m}{ }^{\prime}\right)^{2} / \varepsilon_{m}{ }^{\prime \prime} .
$$

Here, the dielectric constant of the metal $\left(\varepsilon_{\mathrm{m}}\right)$ is shown as $\varepsilon_{\mathrm{m}}{ }^{\prime}+\mathrm{i} \varepsilon_{\mathrm{m}}{ }^{\prime}$.

In case of a single Ta layer, SPP are expected to hardly propagate. $L_{S P P}$ at the metal/air interface was estimated to be about $33 \mu \mathrm{m}$ for a $\mathrm{Ag}$ layer, while the $\mathrm{L}_{\mathrm{SPP}}$ was about $0.05 \mu \mathrm{m}$ for a Ta layer at a wavelength of $630 \mathrm{~nm}$. This implies that $\mathrm{Ag}$ is much closer to an ideal metal than Ta and SPP resonance was therefore estimated to be greatly decreased by the Ta coating.

Figure 4 shows the dependence of the enhancement factor on the number of gratings and the effect of the Ta layer coating on the silver film. Here, the enhancement factor is defined as the relative transmission peak

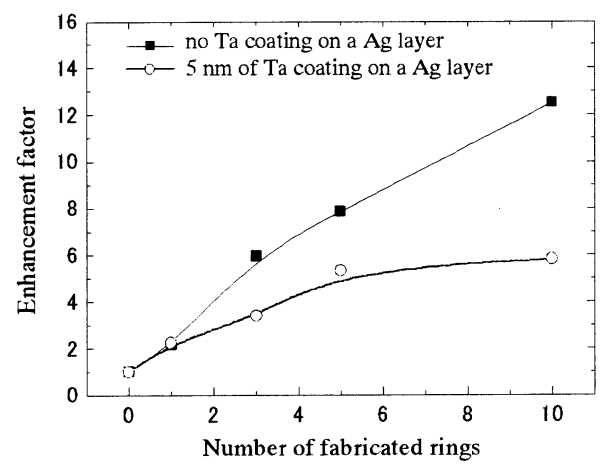

Fig. 4 Dependence of enhancement factor on number of gratings. 


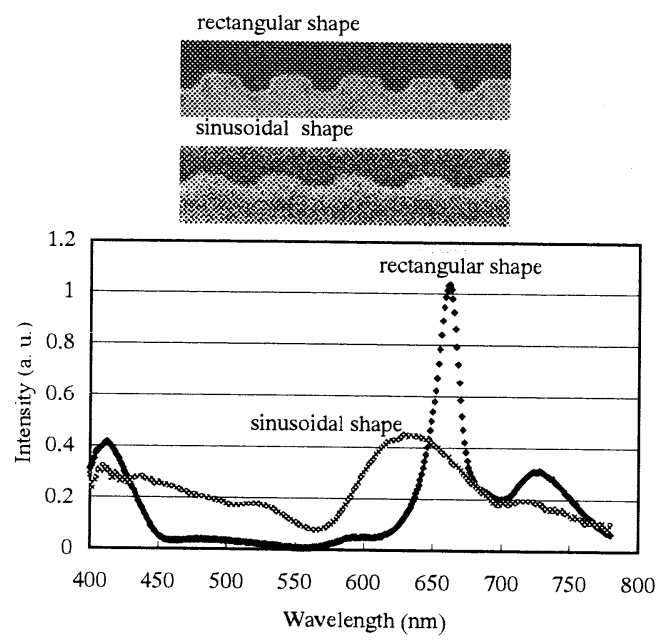

Fig. 5 Transmission light spectra from $150-\mathrm{nm}$ single aperture with re ctangular and round gratings.

intensity to that of a simple hole at the surface plasmon resonant wavelength. The enhancement factor monotonously increases with the number of gratings without the Ta coating. However, for $5 \mathrm{~nm}$ of Ta coated samples, the enhancement factor saturates at about five gratings. This means that surface propagation of SPP is sensitive to the surface optical feature, and it is connected to the optical tunneling effect. The dependence on the number of the gratings is connected to SPP propagation and the focusing lens area both at the in - and exit-sides, and the low-loss optical surface feature of the metal effectively confines energy in the concentric grating metal.

Figure 5 shows the transmission spectra from a $150 \mathrm{~nm}$ single aperture with rectangular and round grating structures. The rectangular grating had a sharper grating edge and excited the SPP mode effectively with smaller periods, and a sharper and a larger transmission peak was obtained. The enhancement factor was about 100 for the rectangular grating.

The transmission efficiency and peak wavelength were also affected by the duty ratio and the grating depth. At a $50 \%$ duty ratio, the largest intensity for the transmission peak was obtained, because SPP excitation between the grating edges was thought to arise strongly in the case of a regular cycle. A deeper grating structure have a larger effective refractive index, therefore, SPP resonance was excited at a lower energy.

By using the optimized grating structure, a power density as large as that of an input laser was obtained for a smaller aperture of $100 \mathrm{~nm}$ diameter.

\subsection{Near-field enhancement effect}

Next, the near-field properties of the bull's eye structure were investigated with NSOM. Figures 6(a) and (b) are topography image and near-field image of light from the subwavelength aperture observed by NSOM. The sample was illuminated by a white light source from a halogen lamp. The near-field intensity was highly enhanced around the aperture position. Figure 6(c) shows the

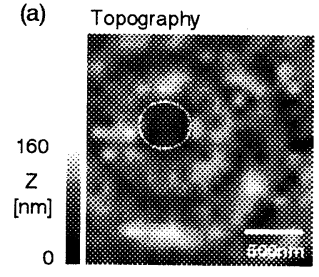

(b) Intensity area : $570-640 \mathrm{~nm}$
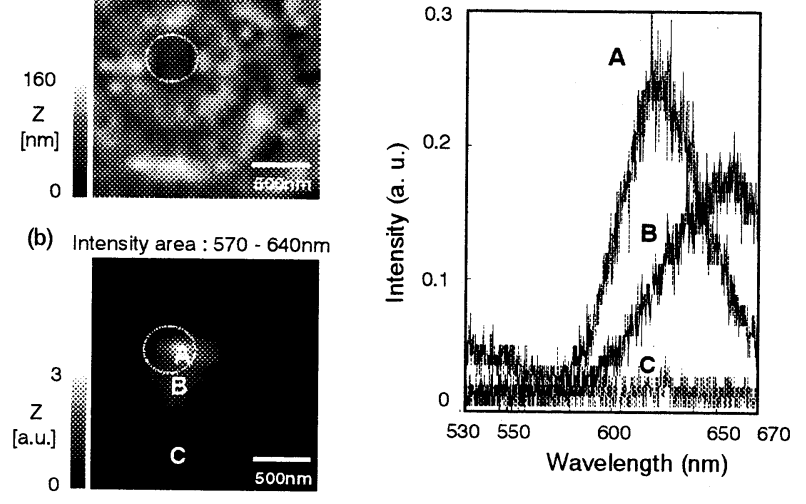

Fig. 6 (a) Topography image and (b) near-field image of light from subwavelength aperture of $150 \mathrm{~nm}$ in diameter by NSOM.

(c) Spectra for near-field light at position A, which is at center position of aperture, at position $B$, which is off-center position around first grating edge, and at position $\mathrm{C}$, which is far from aperture.

spectra for the light at position $A$, which is at the center of the aperture, and at position $B$, which is off center. The spectrum has a sharper peak than that of the far-field spectra. The wavelength of the peak is a little different for $A$ and B. Ebbesen et al. reported the dependence of transmission- light spectroscopy on illuminating-light angles for subwavelength hole arrays, and the role of exit grating in emitting -light dispersion angles. ${ }^{6)}$ That is, the near-field intensity profile differed according to the emission wavelength, and the light of a $630 \mathrm{~nm}$ wavelength was confined around the aperture. For practical purposes, these show that the tuning input -light wavelength of SPP resonance conditions contributes to a sharper spot for the enhanced near-field light.

Figure 7 shows the near-field intensity profile image of light from the subwavelength aperture with a 150-nm diameter. In this experiment, the sample was illuminated by the laser of 630-nm wavelength, which is the spectrum peak wavelength for SPP resonance. As a reference sample, a simple hole sample was also measured by NSOM. The near-field intensity from the single aperture

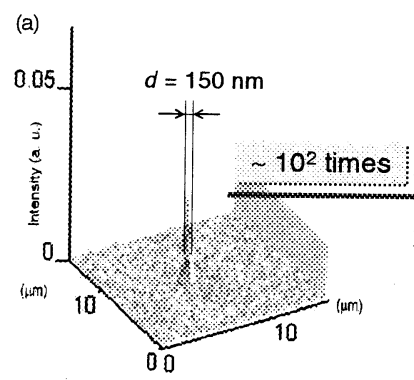

Simple hole (\$150 nm)

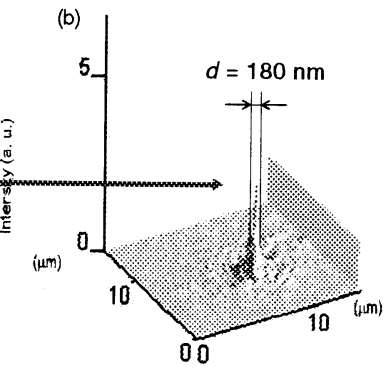

With SP enhancement
Fig. 7 Near-field intensity profile image of light from subwavelength aperture of $150 \mathrm{~nm}$. 
with the concentric grating structure was about 100 times higher than that of the simple aperture. About $180 \mathrm{~nm}$ of near-field light comparable to the aperture size was confirmed. We also observed SPP propagation around the single aperture at the SPP resonance wavelength, which revealed an SPP resonance image on the concentric grating.

\subsection{Near-field optical recording}

We then fabricated a near-field optical head with an SPP resonance structure, and demonstrated near-field optical recording.

Figure 8 shows (a) a schematic drawing of a near-field optical head module, and (b) and (c) micrographs of the fabricated head. The optical components consisted of a single-mode fiber (SMF), two planar microlenses (PML), a dielectric multilayer mirror, and an SPP resonance element, which were arranged on the $\mathrm{SiO}_{2}$ bench. Input light from the laser diode with a wavelength of $630 \mathrm{~nm}$ was introduced by the SMF, collimated by the first PML, deflected by the dielectric multilayer mirror, and focused onto the concentric grating structures by the second PML. The illuminated spot size was designed to be about $4 \mu \mathrm{m}$, the same as the core diameter of the SMF. The aperture diameter of the SPP resonance structure was $100 \mathrm{~nm}$.

Figure 9 shows a micrograph of the head-gimbal assembly, composed of the near-field optical head and the suspension spring. The near-field optical head has an air-bearing surface structure like the conventional magnetic head for hard disk drives, and it was designed to slide over the recording media at a height of no more
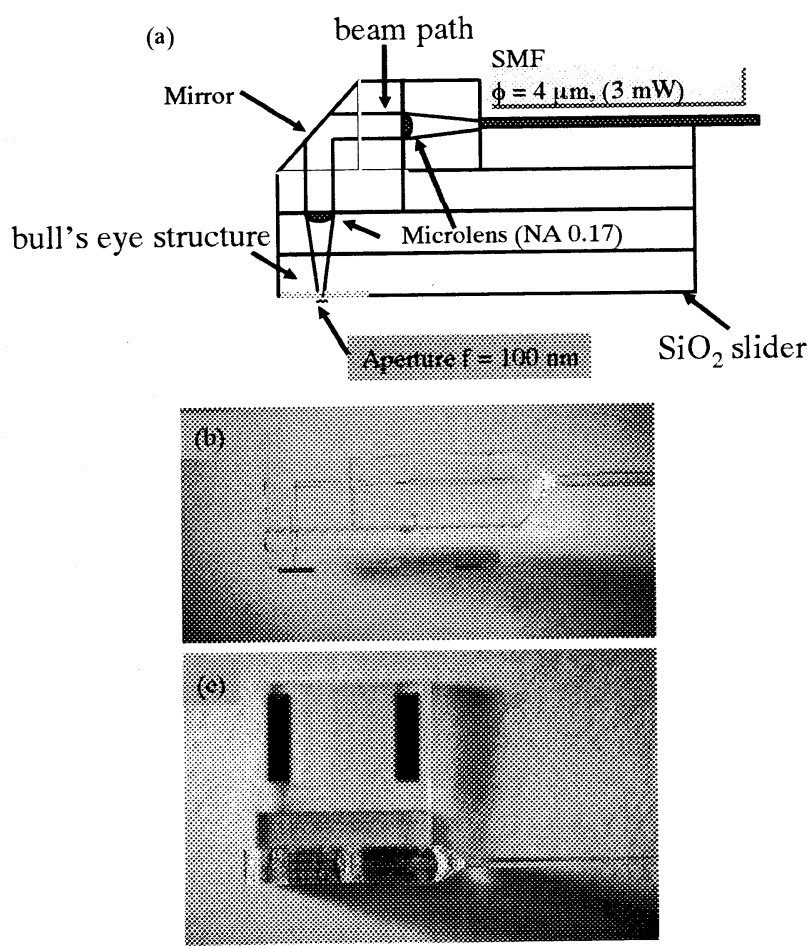

Fig. 8 (a) Schematic drawing of near-field optical head module.

(b), (c) Micrograph of fabricated optical head module.

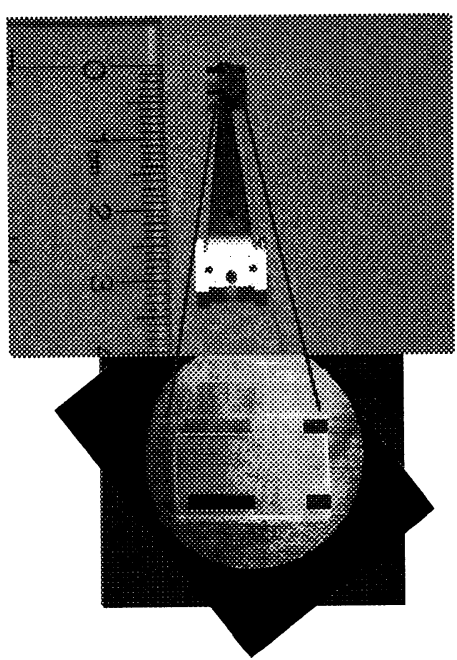

Fig. 9 Micrograph of head-gimbal assembly.

than $50 \mathrm{~nm}$ when the optical recording disc was rotated at $1500 \mathrm{rpm}^{20}$ )

Figures 10(a) and (b) show the topography image and the near-field image of the SPP resonance optical head. A bright spot of about $120-\mathrm{nm}$ in diameter was observed at the center aperture. The throughput power ratio from the $100-\mathrm{nm}$ center aperture was about $1 \%$, and the areal power density was about 100 times enhanced.

Near-field optical recording was evaluated using Ge-Sb-Te phase change media with a GeTe-rich pseudo binary composition. The optical head was scanned over the media with a near-contact mode, using a micro-manipulation system. The head-to-media distance was monitored with an optical interference system.

Figures 11(a) and (b) show near-field recorded pattern images on the phase-change media observed by reflection- mode NSOM. The $500 \mu \mathrm{sec}$ pulse width for the recorded laser was sufficiently long to change the Ge-Sb-Te film from amorphous to crystal. Here, 200-300 $\mathrm{nm}$ of recorded patterns were made on the Ge-Sb-Te phase change media. By optimizing the pulse width to 50 $\mu$ sec, about 100-nm of small patterns comparable to the aperture diameter were formed on the phase change media. Input laser energy to form a small bit on the recording media was much smaller than that previously reported. $^{1)-2)}$

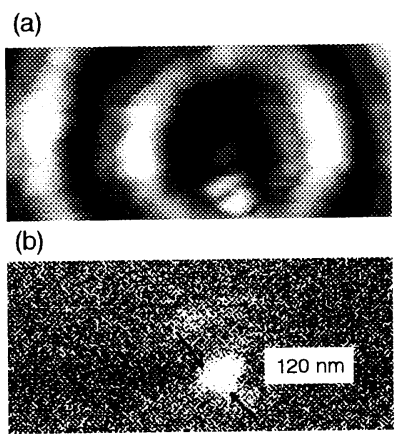

Fig.10 (a) Topography image and (b) near-field image of SPP resonance element by NSOM 
(a)

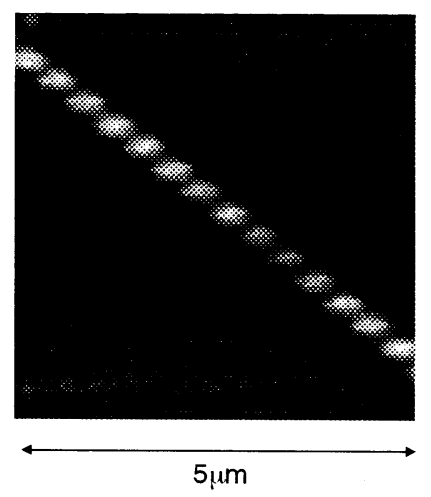

Fig. 11 Near-field recorded pattern image on phase-change media observed by reflection mode NSOM. (a) $500-\mu \mathrm{sec}$ pulse (b) $50-\mu \mathrm{sec}$ pulse with LD power of $3 \mathrm{~mW}$.

These results indicated that remarkably enhanced near-field light was confined around the center aperture and effectively heated the local area of the phase change media by using the bull's eye structure.

\section{Conclusion}

Enhanced photon-tunneling and near-field enhancement effect for a nano-scale aperture with a concentric surface plasmon resonance structure was studied. About two-orders larger light transmission and an enhanced near-field were achieved by optimizing the grating structures. This surface plasmon enhanced near-field light was confined around an aperture with the size comparable to the aperture diameter, which shows this near-field enhancement technique enables us to manipulate a nano-scale light. This enhanced near-field was applied to optical recording, and about $100-\mathrm{nm}$ recorded patterns were successfully made on phase-change media. This surface plasmon optics is expected to play a significant role in future ultra-high-density recording technology.

Acknowledgements The authors wish to thank T. W. Ebbesen for helpful discussion. The authors would also like to thank K. Ichihara of Toshiba Corporation for providing the recording media.

\section{References}

1) E. Betzig, J. K. Trautman, R. Wolfe, E. M. Gyorgy, P. L. Finn, M. H. kryder, and C-H. Chang: Appl. Phys. Lett., 61, 142 (1992).

2) S. Hosaka, T. Shintani, M. Miyamoto, A. Kikukawa, M. Yoshida, K. Fujita, and S. Kammer: J. Appl. Phys., 79, 8082 (1996).

3) T. Yatsui, M. Kourogi, K. Tsutsui, M. Ohtsu, and J. Takahashi: Opt. Lett., 25, 1279 (2000).

4) H. A. Bethe: Phys. Rev., 66, 163 (1944).

5) A. Nahata, R. A. Linke, T. Ishi, and K. Ohashi: Opt. Lett., 28, $423(2003)$.

6) H. J. Lezec, A. Degiron, E. Devaux, R. A. Linke, L. Martin-Moreno, F. J. Garcia-Vidal, and T. W. Ebbesen: Science, 297, 820 (2002).

7) T. Thio, H. J. Lezec, and T. W. Ebbesen: Physica B, 279, 90 (2000)

8) J. Fujikata, T. Ishi, H. Yokota, M. Yanagisawa, and K. Ohashi: Optics Japan 2002, 3pA6 (2002).

9) L. Martin-Moreno, F. J. Garcia-Vidal, H. J. Lezec, A. Degiron, and T. W. Ebbesen: Phys. Rev. Lett., 90, 167401 (2003).

10) F. J. Garcia-Vidal, H. J. Lezec, T. W. Ebbesen, and L. Martin-Moreno: Phys. Rev. Lett., 90, 213901 (2003)

11) W. L. Barnes, A. Dereux, and T. W. Ebbesen: Nature, 424, 824 (2003).

12) L. Martin-Moreno, F. J. Garcia-Vidal, H. J. Lezec, K. M. Pellerin, T. Thio, J. B. Pendry, and T. W. Ebbesen: Phys. Rev. Lett., 86, 1114 (2001)

13) E. Popov, M. Neviere, S. Enoch, and R. Reinisch; Phys. Rev. B, 62, 16100 (2000).

14) P. Lalanne, C. Sauvan, J. P. Hugonin, J. C. Rodier, and P. Chavel: Phys. Rev. B, 68, 125404 (2003).

15) F. J. Garcia-Vidal and L. Martin-Moreno: Phys. Rev. B, 66, 155412 (2002).

16) M. M. J. Treacy: Phys. Rev. B, 66, 195105 (2002).

17) K. Yusu, S. Ashida, N. Nakamura, N. Oomachi, N. Morishita, A. Ogawa and K. Ichihara: Jpn. J. Appl. Phys., 42, 858 (2003).

18) W. L. Barnes, T. W. Preist, S. C. Kitson, and J. R. Sambles: Phys. Rev. B, 54, 6227 (1996).

19) H. Rather, Surface Plasmons on Smooth and Rough Surfaces and on Gratings (Springer-Verlag, Berlin, 1988).

20) M. Yanagisawa, J. Fujikata, T.Ishi, H. Yokota, K. Kato, M. Nakada, K. Ishihara, and K. Ohashi: IIP/ISPS Joint MIPE'03, ORS-09 (2003).

Received May 7; Accepted July 5, 2004 